\title{
Fast near-field far-field transformation for phaseless and irregular antenna measurement data
}

\author{
G. Schnattinger, C. Lopez, E. Kılıç, and T. F. Eibert \\ Lehrstuhl für Hochfrequenztechnik, Technische Universität München, Munich, Germany \\ Correspondence to: G. Schnattinger (georg.schnattinger@tum.de)
}

Received: 3 December 2013 - Revised: 20 January 2014 - Accepted: 29 January 2014 - Published: 10 November 2014

\begin{abstract}
The characterization of antenna radiation patterns by transformed near-field measurements requires accurate amplitude and phase data. This represents a problem since expensive measurement equipment is required, especially at millimeter and submillimeter wavelengths (Isernia et al., 1996). Amplitude-only antenna field measurements are theoretically sufficient for the unique determination of antenna far-fields. Therefore, phaseless techniques are of special interest. However, the required field transformations are extremely challenging, since they are nonlinear and strongly ill-posed.

In this work, the amplitude-only or phaseless near-field far-field transformation problem is formulated as a nonlinear optimization problem. The linear radiation operator within the nonlinear formulation is evaluated using the fast irregular antenna field transformation algorithm (FIAFTA). A hybrid solution procedure is described which combines a genetic algorithm with an iterative conjugate gradient (CG) search method. Numerical results prove the efficiency and flexibility of the formulation and it is shown that the algorithm remains stable when the noise level in the measurements is moderate. Nevertheless, regularization techniques might be beneficial to further improve the robustness of the algorithm.
\end{abstract}

\section{Introduction}

Near-field far-field transformations of antenna measurements with amplitude and phase data are well established (Yaghjian, 1986). The well-known methods for planar, cylindrical and spherical measurements are widely employed in practical applications. These canonical transformation algorithms correspond to solving a linear equation system efficiently by applying the fast Fourier transform (FFT).
Many efforts have been spent to implement phaseless techniques, where only amplitude data is available. Formulating the problem in a general way leads to a nonlinear optimization problem in contrast to the linear one in case of available phase information. Initially, the cost functions were directly based on the amplitude errors in each measurement point. However, using the power mismatch by squaring the amplitudes results in a more favorable nonlinearity (Pierri et al., 1999).

Iterative algorithms with two different techniques have emerged to solve the optimization problem. The first scheme is based on two separate measurement planes, where the fields are alternately propagated from one measurement plane to another one while certain corrections are applied until the fields become stationary. This is referred to as planeto-plane algorithm (Yaccarino and Rahmat-Samii, 1999). The second scheme is based on an iterative optimization approach such as the Gauss-Newton or the conjugate gradient (CG) method (Habashy and Abubakar, 2004; Capozzoli et al., 2009).

Since the solution space of phaseless transformation problems is often populated with local optima (Isernia et al., 1996), the main challenge is to obtain the globally optimal solution. Much research has been performed on this topic and under certain conditions it is possible to guarantee convergence (Pierri et al., 1999). Global optimization strategies such as genetic algorithms have been applied to find the correct solution (Regue et al., 2001). Almost all results have been reported for the fast canonical transformation algorithms which usually limit the applicability to measurements on regular grids.

In this paper, the fast irregular antenna field transformation algorithm (FIAFTA) (Qureshi et al., 2013) is utilized and adapted for the processing of phaseless data. FIAFTA is 
based on multi-level fast multipole method (MLFMM) principles (Chew et al., 2001). It supports arbitrary measurement positions and orientations with full consideration of arbitrary probe characteristics. Since the antenna under test (AUT) can be decomposed using an octree structure, arbitrary information about the shape of the AUT can be included in the transformation as a priori knowledge (Schmidt and Eibert, 2012). Furthermore, it is possible to compute equivalent currents on a surface enclosing the AUT (Eibert et al., 2010). Another advantage is the inherent suppression of truncation errors (Schmidt and Eibert, 2010). Echo suppression techniques are also available to improve the performance in non-anechoic environments (Yinusa and Eibert, 2013). The accuracy typically increases with the number of solver iterations, but in case of measurement uncertainties or errors the opposite can happen. Therefore, aborting the iterative solver after a few iterations can improve the results due to the regularization properties of the iterative procedure (Calvetti et al., 2002). In summary, FIAFTA is more flexible than other methods available at present while its computational efficiency remains high. The topic of this paper is to utilize these advantages for improved transformations of phaseless near-field measurement data by using a CG method in combination with a genetic algorithm.

Section 2 briefly describes the principles of the classical FIAFTA. Section 3 introduces the phaseless inverse problem on top of this by formulating a nonlinear optimization problem together with a suitable solution procedure. Numerical results are given in Sect. 4. Finally, conclusions are drawn in Sect. 5.

\section{Principles of the Fast Irregular Antenna Field Trans- formation Algorithm (FIAFTA)}

As mentioned in the introduction, FIAFTA can be applied to all kinds of amplitude and phase data. The general method is depicted in Fig. 1. The AUT is represented with $N$ equivalent sources and then an inverse problem is formulated using the measurement data. Mathematically speaking this leads to the linear equation system

$\mathbf{A x}=\boldsymbol{b}$,

where $\boldsymbol{b} \in \mathbb{C}^{M}$ contains $M$ complex-valued measurements with phase and amplitude information, $\boldsymbol{x} \in \mathbb{C}^{N}$ is the vector of coefficients which represents the equivalent sources and the matrix $\mathbf{A} \in \mathbb{C}^{M \times N}$ defines the linear relationship between the unknowns and the right hand side. The unknowns $\boldsymbol{x}$ can originate from an equivalent currents representation, a plane wave expansion or a spherical harmonics expansion.

The linear equation system in Eq. (1) is solved by applying an iterative solver such as GMRES or CG to the normal equation

$\mathbf{A}^{\mathrm{H}} \mathbf{A} \boldsymbol{x}=\mathbf{A}^{\mathrm{H}} \boldsymbol{b}$.
This yields the minimum least squares solution $\min \|\mathbf{A} \boldsymbol{x}-\boldsymbol{b}\|_{2}^{2}$. Typically, the residual attains very low values after a few solver iterations and the solution time is dominated by the matrix-vector products with $\mathbf{A}$ and its adjoint $\mathbf{A}^{\mathrm{H}}$. For this reason, it is important to evaluate these products in a fast manner by employing the MLFMM methodology. Also, for the phaseless transformation approach in the next section, it is assumed that the matrix $\mathbf{A}$ can be only evaluated through the matrix-vector products and the entries of the matrix A cannot be accessed directly.

\section{Application of FIAFTA to phaseless near-field data}

\subsection{Problem formulation}

In this section, the phaseless transformation problem will be formulated as a nonlinear optimization problem. Squaring the equation system in Eq. (1) on both sides yields

$\mathbf{A x} \circ(\mathbf{A x})^{*}=\boldsymbol{c}$,

where * denotes the complex conjugate, $\circ$ is the element-wise product between two vectors also referred to as Hadamard product and $\boldsymbol{c}=\boldsymbol{b} \circ \boldsymbol{b}^{*}$ is the vector containing the squared measurement amplitudes. The nonlinear equation system in Eq. (3) can be converted into the nonlinear optimization problem

$\min _{\boldsymbol{x}}\left\|\mathbf{A} \boldsymbol{x} \circ(\mathbf{A} \boldsymbol{x})^{*}-\boldsymbol{c}\right\|_{2}^{2}$.

The measurement data must contain enough information, so that only a single solution will minimize the cost function. In contrast to the linear problem discussed before, the sampling density must be increased at least by a factor of 2 in every spatial dimension to compensate for the missing phase information (Capozzoli et al., 2009). The final solution will be scaled by an arbitrary constant phase factor in comparison to the solution in Eq. (2), because the phaseless measurements do not provide information about the absolute value of the phase.

\subsection{Solving the nonlinear optimization problem}

To solve the nonlinear optimization problem defined in the previous section, a combined solution approach is presented. Nonlinear optimization problems are usually difficult to solve, because they can have many local extrema. Most nonlinear solvers can be classified as one of the two following approaches. On the one hand, iterative solvers are capable of approaching a minimum with great accuracy, but it is very unlikely that the result will correspond to the desired global minimum. On the other hand, global optimization strategies such as genetic algorithms, grid search and simulated annealing are likely to approach the globally optimal solution at some point, but the final result may still not be very accurate. Therefore, it is promising to combine both optimization 

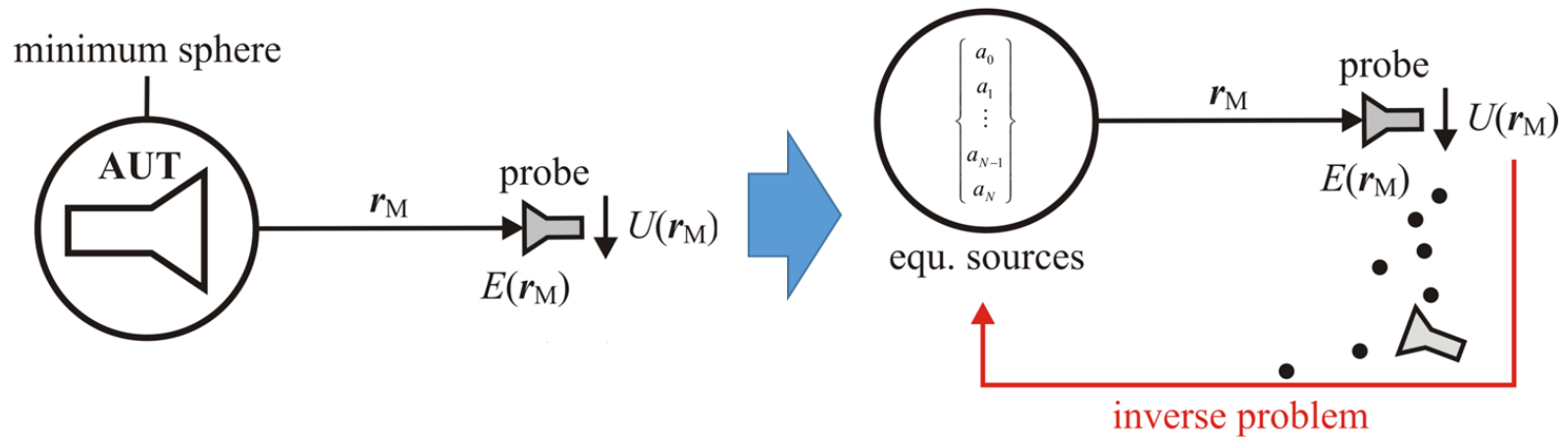

Figure 1. An inverse problem is defined by replacing the AUT by equivalent currents.

approaches. The iterative optimization approach will be explained first. After that, a combined scheme based on a genetic algorithm will be introduced.

Iterative solvers for nonlinear optimization problems are presented in Habashy and Abubakar (2004). First, it is necessary to approximate the function locally using a Taylorseries expansion. Expanding a real cost function with respect to complex arguments requires either a formulation with separated real and imaginary parts or with complex variables and their complex conjugates (Sorber et al., 2012). The latter scheme is employed, because it is computationally more efficient.

By combining the independent variables and their complex conjugates into the vector

$z=\left[\begin{array}{c}x \\ x^{*}\end{array}\right]$

the notation can be written more concisely. The cost function in Eq. (4) is referred to by the symbols

$C(z)=\frac{1}{2}\|\boldsymbol{e}(z)\|_{2}^{2}, \quad \boldsymbol{e}(z)=\mathbf{A} \boldsymbol{x} \circ(\mathbf{A x})^{*}-\boldsymbol{c}$,

where $\boldsymbol{e}$ is the vector of squared measurement errors. Expanding this expression by a Taylor series expansion of second order yields

$C(z) \approx C\left(z_{k}\right)+g\left(z_{k}\right)^{\mathrm{H}} \Delta z+\frac{1}{2} \Delta z^{\mathrm{H}} \mathbf{G}\left(z_{k}\right) \Delta z$,

where $\Delta z=z-z_{k}$ is the offset from the expansion point $z_{k}$,

$\boldsymbol{g}\left(z_{k}\right)=\left.\frac{\partial C(z)}{\partial z}\right|_{z_{k}}=\mathbf{J}\left(z_{k}\right)^{\mathrm{H}} \boldsymbol{e}\left(z_{k}\right)$

is referred to as the gradient vector, $\mathbf{J}\left(z_{k}\right)=\left.\frac{\partial \boldsymbol{e}(z)}{\partial z}\right|_{z_{k}}$ is the Jacobian and $\mathbf{G}\left(z_{k}\right)=\left.\frac{\partial^{2} C(z)}{\partial z \partial z^{\mathrm{H}}}\right|_{z_{k}}$ is the Hessian. Straightforward matrix algebra yields

$\mathbf{J}\left(z_{k}\right)=\left[\left(\operatorname{diag}\left(\mathbf{A} \boldsymbol{x}_{k}\right)\right)^{*} \mathbf{A} \quad \operatorname{diag}\left(\mathbf{A} \boldsymbol{x}_{k}\right) \mathbf{A}^{*}\right]$ for the Jacobian and

$$
\begin{aligned}
\mathbf{G}\left(z_{k}\right)= & \frac{1}{2} \mathbf{J}\left(z_{k}\right)^{\mathrm{H}} \mathbf{J}\left(z_{k}\right)+ \\
& 2\left[\begin{array}{cc}
\mathbf{A}^{\mathrm{H}} \operatorname{diag}\left(\boldsymbol{e}\left(z_{k}\right)\right) \mathbf{A} & \mathbf{0} \\
\mathbf{0} & \mathbf{A}^{\mathrm{T}} \operatorname{diag}\left(\boldsymbol{e}\left(z_{k}\right)\right) \mathbf{A}^{*}
\end{array}\right]
\end{aligned}
$$

for the Hessian (Habashy and Abubakar, 2004), where $\operatorname{diag}($ ) with a vector as argument represents a diagonal matrix. Further simplification is possible by inserting Eqs. (9) and (10) into Eq. (7). Some minor calculations lead to the more computationally efficient terms

$\boldsymbol{g}\left(z_{k}\right)^{\mathrm{H}} \Delta \boldsymbol{z}=2 \boldsymbol{e}\left(z_{k}\right)^{\mathrm{T}} \operatorname{Re}\left(\left(\operatorname{diag}\left(\mathbf{A} \boldsymbol{x}_{k}\right)\right)^{*} \mathbf{A} \Delta \boldsymbol{x}\right)$

and

$$
\begin{aligned}
\Delta \boldsymbol{z}^{\mathrm{H}} \mathbf{G}\left(\boldsymbol{z}_{k}\right) \Delta \boldsymbol{z}=4\left\|\operatorname{Re}\left(\left(\operatorname{diag}\left(\mathbf{A} \boldsymbol{x}_{k}\right)\right)^{*} \mathbf{A} \Delta \boldsymbol{x}\right)\right\|_{2}^{2}+ \\
2 \Delta \boldsymbol{x}^{\mathrm{H}} \mathbf{A}^{\mathrm{H}} \operatorname{diag}\left(\boldsymbol{e}\left(\boldsymbol{z}_{k}\right)\right) \mathbf{A} \Delta \boldsymbol{x},
\end{aligned}
$$

where $\Delta \boldsymbol{x}=\boldsymbol{x}-\boldsymbol{x}_{k}$ is the offset without the complex conjugate.

In the following, the nonlinear CG approach with PolakRibière search directions is employed. It is an iterative gradient search method which requires an initial solution candidate $z_{0}$. The solution candidate is then iteratively refined by

$z_{k+1}=z_{k}+\gamma_{k} s_{k}$

where $z_{k}$ is the previous solution candidate in the $k$-th iteration, $\boldsymbol{s}_{k}$ is the search direction and $\gamma_{k}$ is the step size scaling factor. The search directions are computed by $s_{0}=-\boldsymbol{g}\left(z_{0}\right)$ and

$\boldsymbol{s}_{k}=-\boldsymbol{g}\left(z_{k}\right)+\frac{\boldsymbol{g}\left(z_{k}\right)^{\mathrm{H}}\left(\boldsymbol{g}\left(z_{k}\right)-\boldsymbol{g}\left(z_{k-1}\right)\right)}{\left\|\boldsymbol{g}\left(z_{k-1}\right)\right\|^{2}} \boldsymbol{s}_{k-1}$,

where this definition corresponds to the Polak-Ribière scheme. The expression

$\boldsymbol{g}\left(\boldsymbol{z}_{k}\right)=\left(\mathbf{A}^{\mathrm{H}} \operatorname{diag}\left(\mathbf{A} \boldsymbol{x}_{k}\right)\right)^{*} \boldsymbol{e}\left(\boldsymbol{z}_{k}\right)$ 


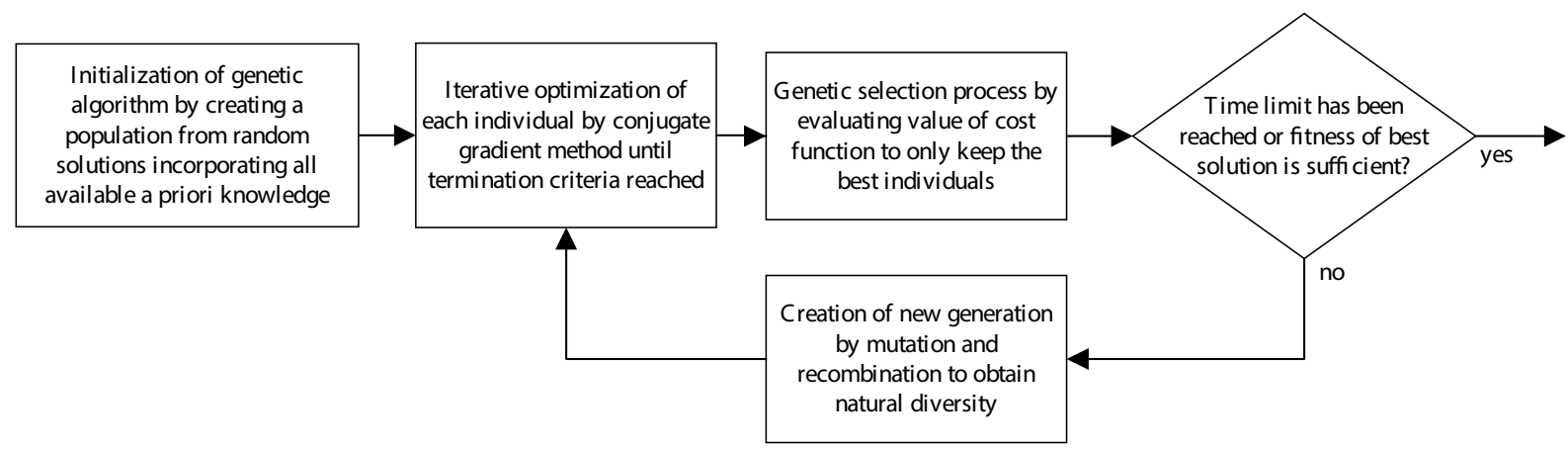

Figure 2. Flow chart of the combined optimization procedure based on a genetic algorithm and a CG method.

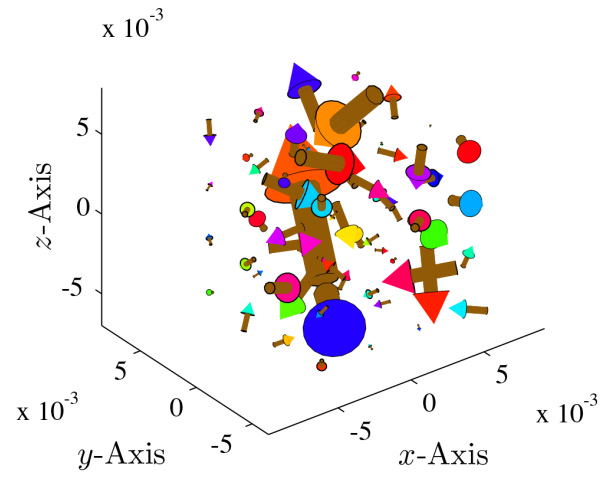

(a) Random configuration of Hertzian dipoles.

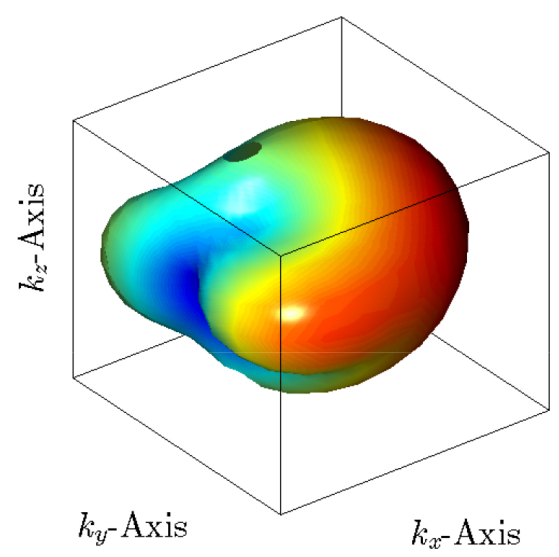

(b) Radiation pattern in the $k$-space domain.

Figure 3. Source configuration used as synthetic AUT for the phaseless near-field far-field transformation.

refers to the gradient vector in iteration $k$ and

$\gamma_{k}=\frac{\left\|\boldsymbol{s}_{k}\right\|_{2}^{2}}{\boldsymbol{s}_{k}^{\mathrm{H}} \mathbf{G}\left(z_{k}\right) \boldsymbol{s}_{k}}$

determines the scaling of the search direction in Eq. (13).

An iterative optimization approach converges rapidly to an extremal value of the cost function. However, the solution is often not desired since it is not equal to the globally opti- mal solution. If an initial solution candidate can be provided which lies in the attraction region of the desired solution, the iterative algorithm computes the correct result. For example, consider a scenario where the measurement equipment provides accurate amplitude information but the phase information is erroneous due to small phase uncertainties. In this case, an approximate solution can be computed by the linear transformation using the inaccurate phase information and subsequently could be used to initialize the nonlinear transformation. The correct result will be obtained if the approximate solution belongs to the attraction region of the desired result which is the case if the phase errors are small enough. In order to achieve convergence to the globally optimal solution, a global optimization scheme is required.

The iterative scheme is now combined with a genetic algorithm. This type of method is inspired from the natural evolution and will be described briefly in the following. Please also consider Fig. 2 for a visualization of the whole optimization scheme. The algorithm starts with an arbitrary set of solution candidates, for example, random solutions. This set is referred to as the population. In the first step, each individual in the population is stimulated by improving it with the iterative optimizer introduced earlier until a certain termination criteria is reached. After this step, all individuals are close to a local optimum or cannot be improved further for some other reason. Then, a selection is performed which identifies the solution with the best fitness. The fitness of an individual is typically obtained by evaluating the cost function. After the selection process, genetic operators such as mutation and recombination are applied. This produces a new generation of individuals which replaces the old one. So the process starts over with the new generation. The overall fitness of the population will gradually improve during the optimization procedure. After some time, the best individual will hopefully be very close to the globally optimal solution. For this to happen, it is sufficient that at some point an individual is created which lies in the attraction region of the globally optimal solution with respect to the given iterative solver. For more information on genetic algorithms, please consider the 

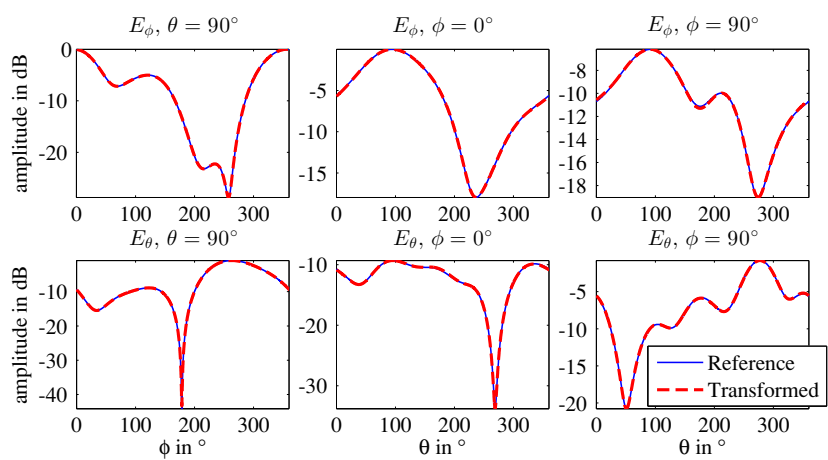

Figure 4. Comparison of reference far-field and transformed nearfield data in various far-field cuts to check accuracy of classical FIAFTA including phase information.

extensive literature which is available on this topic (Regue et al., 2001).

\section{Numerical results}

\subsection{Random configuration of Hertzian dipoles}

In this section, the combined optimization procedure using a genetic algorithm and the CG method will be applied to a synthetic example. A small scenario with random measurements is considered. The width of the AUT is set to $\lambda / 3$ which is $1 \mathrm{~cm}$ at $10 \mathrm{GHz}$. The random dipole distribution depicted in Fig. 3a is used to synthesize the AUT. Figure $3 \mathrm{~b}$ illustrates the resulting far-field pattern in the $k$-space domain. FIAFTA uses 180 spherically sampled plane wave coefficients to represent the unknown radiation characteristics in the transformation. To illustrate the capabilities of FIAFTA and its phaseless extension, 2014 measurements are simulated with a Hertzian dipole probe, where the individual measurements are randomly distributed and oriented between the two spheres with radius $6.5 \mathrm{~cm}$ and $13.5 \mathrm{~cm}$. First, the FIAFTA algorithm is applied to solve the linear problem by exploiting the phase information of the measurements. The transformation result is compared to the reference pattern in Fig. 4 for the three different cuts $\theta=90^{\circ}, \phi=0^{\circ}$ and $\phi=90^{\circ}$ and for both polarizations. It can be observed that the linear transformation result precisely matches the reference solution.

In the second approach, the phase of the measurements is discarded. The genetic algorithm is initialized with 6 random solutions. These random solutions are then optimized with the CG method. The solution with the best fitness is depicted in Fig. 5a. After this step, a selection process is applied where only the three best solutions are kept and seven new individuals are created. Now the CG method is applied to all individuals and the process starts from the beginning. The best individual after a few selected iterations is shown in Fig. 5. In iteration 15 , no visual difference to the reference pattern

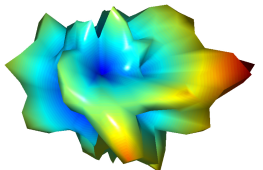

(a) Iteration 1

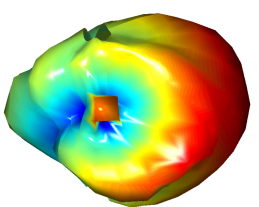

(d) Iteration 9

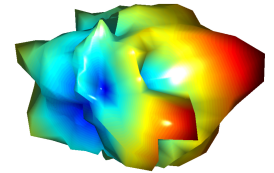

(b) Iteration 2

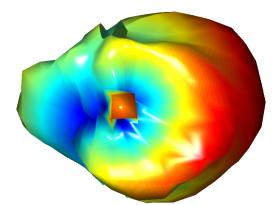

(e) Iteration 13

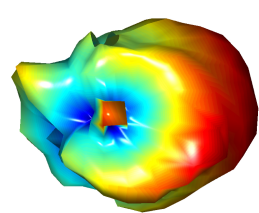

(c) Iteration 4

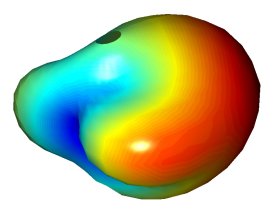

(f) Iteration 15
Figure 5. Phaseless transformation result after various iteration numbers. Reference is given in Fig. 3b.

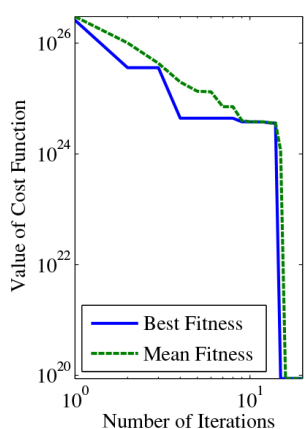

(a) Convergence of genetic algorithm corresponding to the plots in Fig. 5.

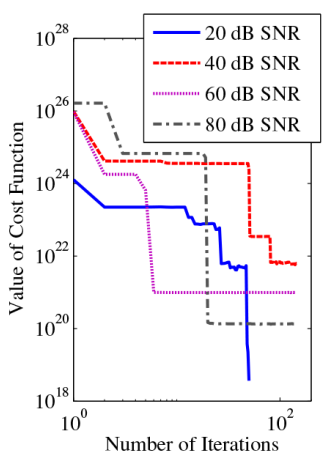

(b) Impact of noise level on the convergence of the phaseless transformation algorithm.
Figure 6. Development of the fitness of the population in the genetic algorithm over the complete runtime.

in Fig. $3 b$ is notable. The gradual improvement of the best individual over all iterations is visualized in Fig. 6a. The blue solid line represents the individual with the best fitness while the dashed line corresponds to the mean fitness of the respective generation. In this example, the described optimization procedure takes about one minute.

In order to test the robustness of the algorithm, white Gaussian noise with different signal-to-noise ratio (SNR) levels was added to the measurement data. In a first experiment, a solution was obtained with the GMRES solver from both the magnitude and phase of the noisy measurements. The solution shows visual distortions using noisy data with an SNR of $20 \mathrm{~dB}$ but starts to visually match the reference pattern from about $30 \mathrm{~dB}$. In this case, the good regularization properties of the GMRES algorithm help to improve the robustness in the linear transformation (Calvetti et al., 2002).

The phaseless transformation was applied to noisy data with an SNR of $20 \mathrm{~dB}, 40 \mathrm{~dB}, 60 \mathrm{~dB}$ and $80 \mathrm{~dB}$. The development of the best fitness over the runtime of the genetic 


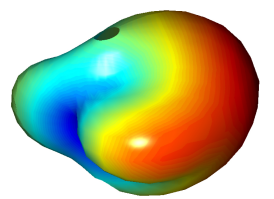

(a) $60 \mathrm{~dB} \mathrm{SNR}$

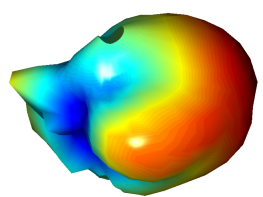

(b) $40 \mathrm{~dB} \mathrm{SNR}$

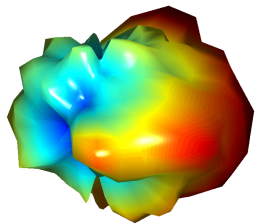

(c) $20 \mathrm{~dB}$ SNR
Figure 7. Impact of noise level on the final solution of the phaseless transformation algorithm.

algorithm is depicted in Fig. 6 for each data set. For $60 \mathrm{~dB}$ and $80 \mathrm{~dB}$ SNR, the algorithm can find the correct solution. However, the noise increases the number of iterations required to find the global optimum and the final value of the cost function increases, too. The final solution for $60 \mathrm{~dB}$ SNR is shown in Fig. 7a and no error is visible. When the level of noise is increased to $40 \mathrm{~dB}$, the solution starts to deviate from the reference pattern as depicted in Fig. 7b. Figure 6 shows that also a larger number of iterations is required. Further increasing the noise level to $20 \mathrm{~dB}$ produces the pattern in Fig. 7c. In contrast to the other results, this solution is obtained very rapidly and the the cost function has an extremely low value. This can only be explained by the fact that the cost function promotes undesirable solutions. Noisy measurement data influences the cost function in a way that the desired solution does not correspond to the globally optimal solution anymore. This is expected, where it should be kept in mind that $20 \mathrm{~dB}$ noise level is of course a rather bad value. Nevertheless, the application of regularization principles in the cost function might be desirable in the future in order to further improve the robustness. For example, it would be possible to penalize the amplitude of the solution vector or an error weighting matrix in the cost function might improve the situation. Moreover, it is always recommended to include as much information as possible about the AUT into the formulation.

In summary, it was shown that it is possible to retrieve an unknown far-field pattern from arbitrarily oriented and located near-field measurements without additional a priori knowledge by applying a nonlinear optimization approach on top of FIAFTA. By further refinement, the methodology will be applicable to larger problems.

\subsection{Robustness analysis involving a single dipole source}

The preceding example has revealed that the robustness of phaseless transformation approaches is an important issue. In the following, this insight should be underpinned by quantitative conclusions.

Since a quantitative analysis typically requires many simulation runs, it is reasonable to choose a simple scenario for this purpose. The selected source configuration and its radiation characteristic is shown in Fig. 8. A single dipole situated at the origin is radiating in free space. Measurement data is

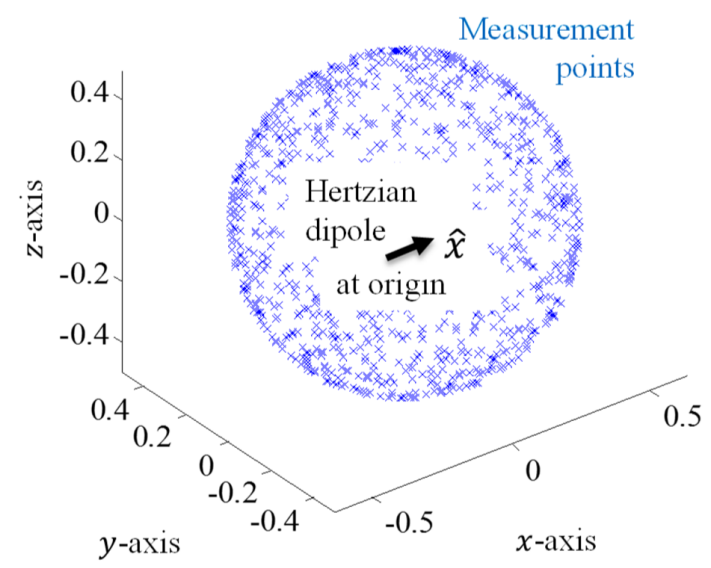

(a) Source surrounded by 1000 randomly located spherical measurements points.

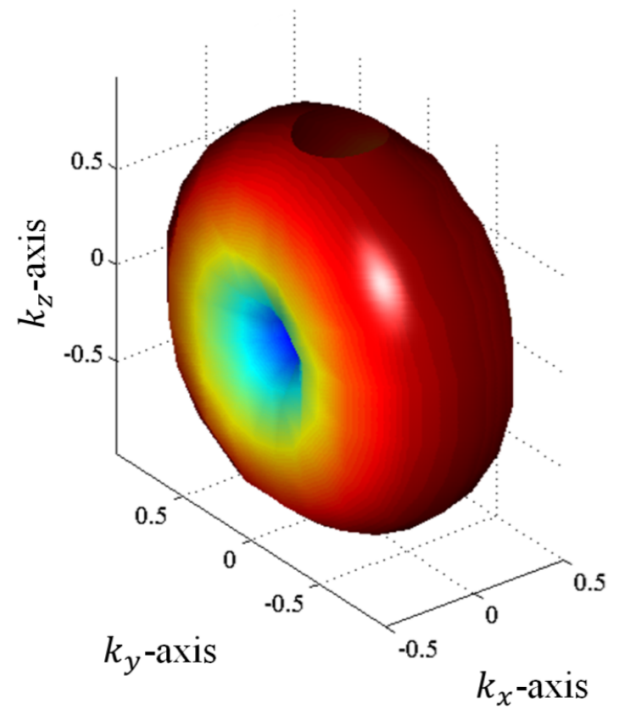

(b) Radiation pattern in the $k$-space domain.

Figure 8. Source configuration consisting of an $\hat{x}$-oriented Hertzian dipole.

generated by randomly distributing 1000 field observations over a sphere with the radius $1.6 \lambda$.

In order to compare the performance of the phaseless transformation with the conventional approach incorporating phase information, several transformation runs have been performend for different levels of measurement noise while varying the number of available measurements from 3 to 1000. The noise level ranging from $-20 \mathrm{~dB}$ to $80 \mathrm{~dB}$ SNR is defined with respect to the mean energy of these measurements.

By using vector spherical harmonics of the first order as equivalent currents, the transformation problem is well defined. Figure 9 summarizes the final results obtained with the nonlinear (phaseless) and the linear (amplitude and phase) approach. Each transformation result is assigned to one of three error levels by rating the visual appearance of the 
Linear Transformation

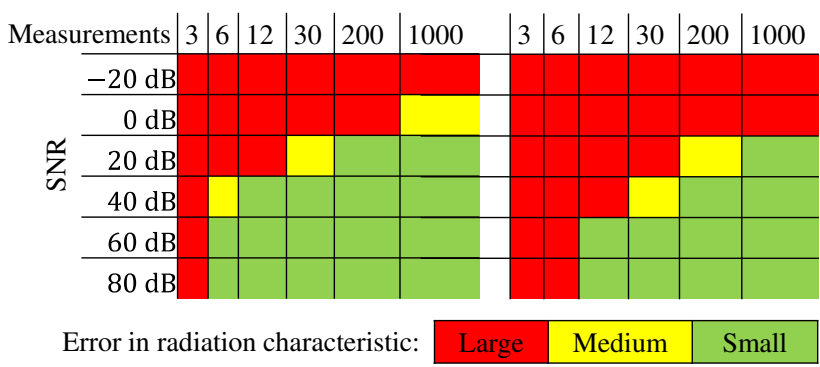

Figure 9. Comparison of phaseless transformation approach and conventional method incorporating phase information with respect to robustness issues regarding measurement noise and measurement redundancy.

reconstructed patterns. The categories 'large', 'medium' and 'small' represent the amount of visible distortion in comparison with the reference pattern in Fig. 8b. By comparing the statistics from the linear and nonlinear transformation approach, the following conclusions are derived: To achieve comparable accuracy, the phaseless transformation approach requires five times more measurements, or alternatively, measurements which are $20 \mathrm{~dB}$ better with respect to the SNR. Be aware that these conclusions are not valid for larger scenarios, because the situation is expected to become worse due to local minimum problems. It will remain a topic of further research to find out how exactly the robustness is affected by the electrical size of the problem.

\section{Conclusions}

Conventional field transformation approaches are not able to process irregularly sampled measurement data without phase information. In order to overcome this limitation, a nonlinear optimization procedure is combined with the fast irregular antenna field transformation algorithm (FIAFTA). The resulting formulation is outstandingly efficient and flexible. However, the nonlinear optimization problem turns out to be very challenging. Good results were achieved using a hybrid solution procedure. It combines a genetic algorithm with an iterative conjugate gradient (CG) search method. Numerical results showed that the algorithm remains stable when the noise level is moderate. Nevertheless, regularization techniques might be beneficial to further improve the robustness of the algorithm.

Edited by: U. van Rienen

Reviewed by: two anonymous referees

\section{References}

Calvetti, D., Lewis, B., and Reichel, L.: On the Regularizing Properties of the GMRES Method, Numer. Math., 91, 605-625, 2002.

Capozzoli, A., Curcio, C., D’Elia, G., and Liseno, A.: Phaseless Antenna Characterization by Effective Aperture Field and Data Representations, IEEE Trans. Antennas Propag., 57, 215-230, doi:10.1109/TAP.2008.2009647, 2009.

Chew, W. C., Jin, J.-M., Michielssen, E., and Song, J.: Fast and Efficient Algorithms in Computational Electromagnetics, Artech House, 931 pp., 2001.

Eibert, T. F., Ismatullah, Kaliyaperumal, E., and Schmidt, C. H.: Inverse Equivalent Surface Current Method with Hierarchical Higher Order Basis Functions, Full Probe Correction and Multilevel Fast Multipole Acceleration (Invited Paper), Prog. Electromagn. Res., 106, 377-394, 2010.

Habashy, T. M. and Abubakar, A.: A General Framework for Constraint Minimization for the Inversion of Electromagnetic Measurements, Prog. Electromagn. Res., 46, 265-312, 2004.

Isernia, T., Leone, G., and Pierri, R.: Radiation Pattern Evaluation from Near-Field Intensities on Planes, IEEE Trans. Antennas Propag., 44, 701-710, 1996.

Pierri, R., D’Elia, G., and Soldovieri, F.: A Two Probes Scanning Phaseless Near-Field Far-Field Transformation Technique, IEEE Trans. Antennas Propag., 47, 792-802, doi:10.1109/8.774132, 1999.

Qureshi, M. A., Schmidt, C. H., and Eibert, T. F.: Near-Field Error Analysis For Arbitrary Scanning Grids Using Fast Irregular Antenna Field Transformation Algorithm, Prog. Electromagn. Res. B, 48, 192-220, 2013.

Regue, J.-R., Ribo, M., Garrell, J.-M., and Martin, A.: A Genetic Algorithm based Method for Source Identification and Far-Field Radiated Emissions Prediction from Near-Field Measurements for PCB Characterization, IEEE Trans. Antennas Propag., 43, 520-530, doi:10.1109/15.974631, 2001.

Schmidt, C. H. and Eibert, T. F.: Near-Field to Far-Field Transformation utilising Multilevel Plane Wave Representation for Planar and Quasi-Planar Measurement Contours, IET Microw. Antenna. P., 4, 1829-1837, 2010.

Schmidt, C. H. and Eibert, T. F.: Sub Spectra Representation of Antennas for Plane Wave Based Near-Field Far-Field Transformation at Short Measurement Distances, IEEE Antennas and Propagation Society International Symposium (AP-S), Chicago, IL, USA, July 2012.

Sorber, L., Barel, M. V., and Lathauwer, L. D.: Unconstrained Optimization of Real Functions in Complex Variables, SIAM J. Optimiz., 22, 879-898, 2012.

Yaccarino, R. and Rahmat-Samii, Y.: Phaseless Bi-Polar Planar Nearfield Measurements and Diagnostics of Array Antennas, IEEE Trans. Antennas Propag., 47, 574-583, doi:10.1109/8.768794, 1999.

Yaghjian, A. D.: An Overview of Near-Field Antenna Measurements, IEEE Trans. Antennas Propag., 34, 30-45, 1986.

Yinusa, K. and Eibert, T.: A Multi-Probe Antenna Measurement Technique with Echo Suppression Capability, IEEE Trans. Antennas Propag., 61, 5008-5016, doi:10.1109/TAP.2013.2271495, 2013. 